\title{
Maternal Outcome among Women Having Cardiac Disease at Tertiary Care Hospital
}

\author{
SHAZIA AWAN ${ }^{1}$, SAIRA DARS ${ }^{2}$, SHAZIA RANI ${ }^{3}$, RAHEEL SIKANDAR $^{4}$ \\ ${ }_{1,2}$ Assistant Professors, ${ }^{3}$ Associate Professor, ${ }^{4}$ Professor, \\ Department of Obstetrics \& Gynaecology, Liaquat University of Medical \& Health Sciences Jamshoro \\ Correspondence to Dr. Shazia Awan E-mail: shaziaasad38@gmail.com Cell: 0333-53655867
}

\begin{abstract}
Aim: To determine the maternal outcome among women presented with cardiac disease at tertiary care hospital. Study design: Prospective study.

Place and duration of study: Department of Obstetrics \& Gynaecology, Liaquat University of Medical and Health Science Jamshoro from $1^{\text {st }}$ April 2019 to $31^{\text {st }}$ March 2020.

Methodology: Fifty-seven women after 24 weeks of alive pregnancy and having cardiac diseases were included. The subjects were seen in the Gynaecology OPD every two weeks till 34-36 weeks, as per institutional protocols and after that every week by obstetrician. Cardiologists saw subjects in each trimester and delivery schedule were developed and medications were adapted with collaboration. The anaesthetic scheme of the subject was also reviewed with the cardiac anaesthetist. All the maternal outcome data was recorded.

Results: Most of the patients $89.5 \%$ had age group of $25-32$ years. Multiparous women were commonest $43.3 \%$ and $53.7 \%$ patients underwent normal vaginal delivery. $35.8 \%$ patients had congenital heart disease, $32.8 \%$ had cardiomyopathy and $16.4 \%$ had Rheumatic heart disease. According to the maternal outcome anemia was most common among $85.1 \%$, pre-eclampsia $41.8 \%$, pulmonary edema $35.8 \%$, IUGR $20.9 \%$, birth asphyxia $26.9 \%$, preterm $38.8 \%$ and maternal mortality $14.9 \%$. Abruption placenta and preterm birth were significantly associated with congenital heart disease and cardiomyopathy $p=0.047$ and $p=0.002$ respectively. Pulmonary edema was higher among patients of cardiomyopathy $(p=0.027)$. Birth asphyxia was higher among patients of rheumatic heart disease. While anemia, IUGR and maternal mortality were equally found among cardiac diseases, $p$-values were quit insignificant.

Conclusion: Cardiac disease showed adverse maternal outcome during pregnancy with mortality rate of $14.9 \%$. Keywords: Pregnancy cardiac disease, Maternal outcome, Mortality rate
\end{abstract}

\section{INTRODUCTION}

Cardiovascular disease is regarded among the major issues of maternal mortality and morbidity in the postnatal and antenatal periods ${ }^{1,2}$. Taking care of cardiac mothers and children is extremely difficult, with problems such as late diagnosis and also insufficient facilities and drug supply ${ }^{1}$. Cardiac disorder in pregnancy represents a spectrum of etiologies, inclusive of cardiomyopathies, valvular HD (VHD), pulmonary hypertension (PH), and adult congenital $\mathrm{HD}^{3}$. Cardiac disease may cause of anemia during pregnancy in $17(3.7 \%) .^{2}$ There are many clinical studies published on the data based on registries like ROPAC registry of pregnant cardiac patients ${ }^{4,5}$.

Rheumatic heart condition is regarded as among the most prevalent forms in developing nations, while in developed nations, congenital heart disease (CHD) and cardiomyopathies are the primary types. ${ }^{6}$ Roughly 0.2 to $2 \%$ of all pregnant females are disturbed by cardiacabnormalities and maternal mortality of $20.5 \%$ is correlated with heart disease. 1,6,7 Vietnam is identified as a lowmiddle-income nation, where agriculture is the primary source of economy. Vietnam's population has risen by 1.07 percent in a year, approaching a total of 93,700,000 individuals, as per statistics of Vietnam's General Department of Population in early 2018. The maternal mortality ratio in 2015 was 54 per 0.1 million live births, and

Received on 12-12-2020

Accepted on 21-04-2021 there were 18 thousand neonatal deaths. ${ }^{1}$ In particular, acquired conditions are rheumatic with involvement of the mitral valve and coronary artery disease. Cardiac muscle disease, particularly cardiomyopathy peripartum, is a serious condition that occurs during postpartum period and pregnancy. It has a high mortality rate ${ }^{8}$.

To encourage ideal care throughout pregnancy that contributes significantly in the outcome, it is vital to carefully assess subjects for underlying cardiac disease. ${ }^{9}$ Management of heart disease during pregnancy is challenging. So heart disease with pregnancy should be managed as team approach involving an obstetrician and cardiologist and other specialists such as anesthesiologist ${ }^{10}$ Furthermore, no official publishing data is accessible for maternal outcomes among pregnant females of Vietnamese with heart disease ${ }^{1}$. It is apparent that fetal development limitation affects $3 \%-7 \%$ of all neonates, which is correlated with several negative outcomes such as stillbirth. ${ }^{1,11}$ This study has been conducted to assess the maternal outcome among women presented with cardiac disease at tertiary care Hospital.

\section{MATERIALS AND METHODS}

This prospective study was conducted at Department of Obstetrics \& Gynaecology, Liaquat University of Medical and Health Science Jamshoro from $1^{\text {st }}$ April 2019 to $31^{\text {st }}$ March 2020. All the women after 24 weeks of alive pregnancy and having cardiac diseases were included. Women with renal failure, diabetes mellitus, chronic liver 
disease, Uremia, eclampsia and pulmonary disease were excluded. Women underwent complete medical history, clinical examination and required laboratory investigations. As per institutional protocol, patients were seen in the Gynaecology OPD every two weeks up to 34-36 weeks and after which by obstetrician every week. Cardiologists saw subjects in each trimester and delivery schedule was developed and medications were adapted with collaboration. The anesthetic scheme of the subject was also shared with the anesthetist of the heart. Date of the $\mathrm{C}$ section and mode of delivery were considered. Delivery was carried out in a specific labor ward of gynecology/obstetrics. Continuous monitoring of blood pressure, oxygen saturation and pulse was performed. Intermittent fetal cardiac rate was monitored by cardiotocograph (CTG) performed in perspective of the high-risk scenario. A 24-hour functional adult cardiac theatre in close vicinity on that same ground helped to quickly manage all complications. Full services for cardiac resuscitation were accessible in the ward together with a resident cardiologist. The data regarding maternal outcome was collected and recorded. Data was analyzed using SPSS version 20.

\section{RESULTS}

Most of the patients $89.5 \%$ had age group of 25-32 years, while remaining $10.5 \%$ patients were with age group of 3340 years and $43.8 \%$ patients had hypertension. Multiparous women were commonest $50.9 \%$, followed by $29.9 \%$ grand multipara and $19.2 \%$ were primipara. Most of the patients 45.7 underwent normal vaginal delivery, 36.8\% underwent C-section, while $17.5 \%$ delivered by instrumental assessment. Majority of patients was un-booked $63.2 \%$ and $36.8 \%$ were booked (Table 1 ).

Table 1: Demographic characteristics of patients $(n=57)$

Table 1: Demographic characteristics of patients (n=57)
\begin{tabular}{|l|c|c|}
\hline Variable & No. & $\%$ \\
\hline Age (years) & 51 & 89.5 \\
\hline $25-32$ & 6 & 10.5 \\
\hline 33.40 & 25 & 43.8 \\
\hline Hypertension & 32 & 56.2 \\
\hline Yes & 29 & 50.9 \\
\hline No & 11 & 19.2 \\
\hline Parity & 17 & 29.9 \\
\hline Multi & \multicolumn{2}{|l|}{} \\
\hline Primipara & 26 & 45.7 \\
\hline Grand multi & 10 & 17.5 \\
\hline Mode of delivery & 21 & 36.8 \\
\hline NVD & 21 & 36.8 \\
\hline Instrumental & 36 & 63.2 \\
\hline C- section & \multicolumn{2}{|l|}{} \\
\hline Booking status & Booked &
\end{tabular}

Table 2: Frequency of cardiac disease $(n=57)$

\begin{tabular}{|c|c|c|}
\hline Cardiac disease & No. & $\%$ \\
\hline \multicolumn{3}{|c|}{ Congenital heart disease } \\
\hline Yes & 24 & 42.1 \\
\hline No & 33 & 57.9 \\
\hline \multicolumn{3}{|c|}{ Rheumatic heart disease } \\
\hline Yes & 11 & 19.3 \\
\hline No & 46 & 80.7 \\
\hline \multicolumn{3}{|l|}{ Cardiomyopathy } \\
\hline Yes & 22 & 38.6 \\
\hline No & 35 & 61.4 \\
\hline
\end{tabular}

Table 3: Frequency of maternal outcome $(n=57)$

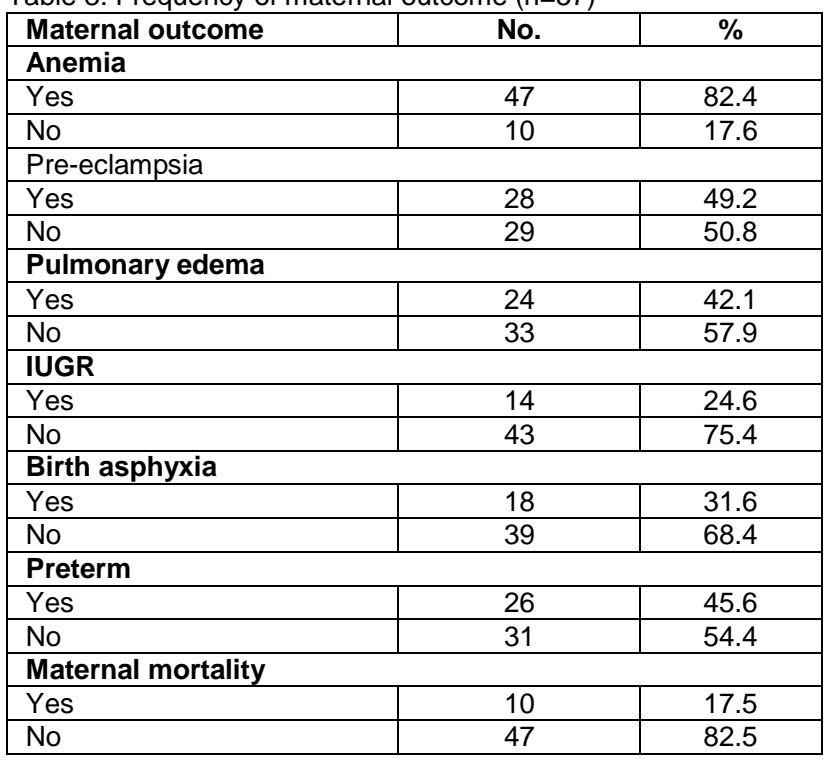

According to cardiac diseases, $42.1 \%$ had congenital heart disease, $38.6 \%$ had cardiomyopathy and $19.3 \%$ had rheumatic heart disease (Table 2).

Maternal outcome anemia was most common among $82.4 \%$ patients, pre-eclampsia developed among $49.2 \%$ patients, pulmonary edema found among $42.1 \%$ patients, IUGR was recorded in $24.6 \%$ patients, birth asphyxia among $31.6 \%$, preterm were $45.6 \%$ and maternal mortality was $17.5 \%$ (Table 3 ).

Abruption placenta and preterm birth were significantly associated with congenital heart disease and cardiomyopathy $(p=0.047$ and $p=0.002)$ respectively. Pulmonary edema was higher among patients of cardiomyopathy $(p=0.027)$. Birth asphyxia was higher among patients of rheumatic heart disease. While anemia, IUGR and maternal mortality were equally found among cardiac diseases, p-values were quite insignificant (Table 4).

Table 4: Maternal outcome according to cardiac diseases $(n=57)$

\begin{tabular}{|l|c|c|c|c|}
\hline \multirow{2}{*}{ Maternal outcome } & \multicolumn{2}{|c|}{ Cardiac Disease } & \multirow{2}{*}{ P value } \\
\cline { 2 - 5 } & Congenital heart disease & Rheumatic heart disease & Cardiomyopathy & 0.113 \\
\hline Anemia & 21 & 11 & 22 & 0.047 \\
\hline Abruption placenta & 7 & 0 & 7 & 0.112 \\
\hline Pre-eclampsia & 14 & 7 & 12 & 0.027 \\
\hline Pulmonary edema & 4 & 4 & 3 & 0.270 \\
\hline IUGR & 4 & 4 & 9 & 0.024 \\
\hline Preterm & 11 & 0 & 7 & 0.002 \\
\hline Birth asphyxia & 3 & 8 & 6 & 0.150 \\
\hline Maternal mortality & 4 & 0 & & 9 \\
\hline
\end{tabular}




\section{DISCUSSION}

Pregnant women with underlying heart disease (HD) are at increased risk for adverse maternal, obstetric, and neonatal outcomes. ${ }^{3}$ In this study most of the patients $89.5 \%$ had age group of $25-32$ years, while remaining $10.5 \%$ patients were with age group of 33-40 years. Comparable findings found in the study of Nguyen Manh et al ${ }^{1}$ majority of patients $86.97 \%$ was under 35 years and only $13.03 \%$ were more than 35 years according to age. Ansari et $\mathrm{al}^{8}$ stated that mean age of women was 28.3 years. Abbasi et $\mathrm{al}^{10}$ reported that $63.38 \%$ patients had age group of $21-25$ years. Pujitha et al ${ }^{11}$ reported that most of the women $50.0 \%$ were with age group of $21-25$ years. In this study on patient's distribution according to cardiac diseases, $35.8 \%$ had congenital heart disease, $32.8 \%$ had cardiomyopathy and $16.4 \%$ had rheumatic heart disease. Owens et $\mathrm{al}^{3}$ reported that $17 \%$ patients had cardiomyopathy, $40 \%$ presented with VHD, 35\% patients had ACHD, and $\mathrm{PH}$ found among $8 \%$. Ansari et $\mathrm{al}^{8}$ reported that the according to cardiac diseases rheumatic heart disease was $70 \%$, congenital heart disease was among $12.4 \%$, arrhythmias in $9.6 \%$ patients, cardiomyopathy was in $6.8 \%$ patients and pulmonary hypertension was only among $1.4 \%$ patients. Abbasi et $\mathrm{al}^{10}$ reported that $76.05 \%$ women had rheumatic heart disease, $15.49 \%$ had CHD and 6 patients had arrhythmia. Pujitha et $\mathrm{al}^{11}$ reported that mostly women had rheumatic heart disease $62.6 \%, 21.8 \% \mathrm{CHD}$ and $15.6 \%$ peripartum cardiomyopathy. In this series according to the maternal morbidity, anemia was most common among $85.1 \%$ patients, pre-eclampsia developed among $41.8 \%$ patients, pulmonary edema found among 35.8\% patients, IUGR was recorded in $20.9 \%$ patients, birth asphyxia among $26.9 \%$ and preterm were $38.8 \%$. Pujitha et $\mathrm{al}^{11}$ found anaemia $45.6 \%$, preterm labor $27.3 \%$, preeclampsia $18.1 \%$, abruptio placentae $9 \%$, pulmonary oedema $4.5 \%$ and pulmonary arterial hypertension $4.5 \%$. Behera et $\mathrm{al}^{12}$ observed that the commonest complications were anaemia $66.6 \%$ preterm labour $22.7 \%$, preeclampsia $16.65 \%$, abruptio placentae $25 \%$.

In this study, maternal mortality was $14.9 \%$. While in the study of Ansari et al ${ }^{8}$ found mate mortality $2.7 \%$. Pujitha et $\mathrm{al}^{11}$ also found maternal mortality $3.12 \%$. Campanharo et $\mathrm{al}^{13}$ found maternal mortality rate $4.8 \%$. Maternal mortality was higher in our study, this may because small sample size and severity of disease.

In the current study, multiparous women were commonest $43.3 \%$. Manh Manh et $\mathrm{al}^{1}$ reported that gravida 3 were only $9.86 \%$ and more were $42 \%$. Behera et al $^{12}$ also observed that $50 \%$ were primigravida, $36.3 \%$ were second gravida and $13.3 \%$ were gravid 3 or more.

Most of the patients 53.75 underwent normal vaginal delivery, $31.3 \%$ underwent C-section, while $14.9 \%$ delivered by instrumental assessment. Majority of patients was un-booked $68.7 \%$ and $31.3 \%$ were booked. Similarly, Behera et $\mathrm{al}^{12}$ found that the labour was spontaneous in onset in all patients. In all patients with vaginal delivery outlet forceps and ventuse applied to cut short the second stage of labour to reduce maternal distress. Pujitha et $\mathrm{al}^{11}$ also found comparable findings as most of patients had vaginal delivery $(81.25 \%)$ and caesarean section was done in 6 patients $(18.75 \%)$.

\section{CONCLUSION}

The heart disease during pregnancy linked to significant maternal morbidity and mortality. Best cardiac management prior to pregnancy can be shoed better pregnancy outcome. A team approach for optimal management is necessary.

\section{REFERENCES}

1. Nguyen Manh T, Bui Van N, Le Thi H, Vo Hoang L, Nguyen $\mathrm{Si}$ Anh $\mathrm{H}$, et al. Pregnancy with heart disease: maternal outcomes and risk factors for fetal growth restriction. Int $\mathrm{J}$ Envir Res Public Health 2019;16(12):2075.

2. Koutrolou-Sotiropoulou P, Parikh PB, Miller C, Lima FV, Butler J, Stergiopoulos K. Impact of heart disease on maternal and fetal outcomes in pregnant women. Am J Cardiol 2015; 116: 474-80.

3. Owens A, Yang J, Nie L, Lima F, Avila C, Stergiopoulos K. Neonatal and maternal outcomes in pregnant women with cardiac disease. J Am Heart Assoc 2018; 7(21): e009395.

4. Humayun $S$, Sultana $R$, Korejo $R$, Ashraf $T$, Jabeen $S$, Yasmin H. Maternal Mortality in Pakistan: An Attention Seeking Problem. J Soc Obstet Gynaecol Pak 2017; 7(1):227.

5. Ruys TPE. Heart failure in pregnant women with cardiac disease: data from ROPAC. Heart 2014; 100:231-8.

6. Mishra R. Ian Donald's practical obstetric problems; New Delhi: BI Publications; 2010.

7. Sawhney H, Aggarwal N, Suri V, Vasishta K, Sharma $Y$, Grover A. Maternal and perinatal outcome in rheumatic heart disease. Int J Gynecol Obstet 2003, 80, 9-14.

8. Ansari A, Khalil H, Javed R, Pervaiz F. Managing pregnant cardiac patients in a tertiary cardiac care unit improves fetomaternal outcome. Pak Armed Forces Med J 2015; 65(Suppl): S75-80

9. Fathy FT, Gonied AS, Mohammed NS. Maternal and neonatal outcome in women with cardiac diseases and suggested nursing guidelines. JNHS 2018;7;2;80-91.

10. Abbasi S, Siddiqua SF, Rijvi S, Akhtar S, Haque B, Jesmin S. Study of maternal and fetal outcome in pregnancy with heart disease. Anwer Khan Modern Med Coll J 2017; 23;8(2):112.

11. Pujitha KS, Sheela SR, Jyothi NS. A study of maternal and fetal outcome in cardiac disease in pregnancy at tertiary care center. Int J Reprod Contracept Obstet Gynecol 2017; 6:5095-8.

12. Behera R, Moharana JJ. Maternal and fetal outcome in cardiac disease in pregnancy: a retrospective study at tertiary care center. Int J Reprod Contracept Obstet Gynecol 2018; 7:4399-402

13. Campanharo FF, Cecatti JG, Haddad SM, Parpinelli MA, Born D, Costa ML, Mattar R, Brazilian Network for Surveillance of Severe Maternal Morbidity Study Group. The impact of cardiac diseases during pregnancy on severe maternal morbidity and mortality in Brazil. PLoS One. 2015 Dec 9;10(12):e0144385. 